\title{
Entanglement of Multipartite Schmidt-correlated States
}

\author{
Ming-Jing Zhao ${ }^{1}$, Shao-Ming Fei ${ }^{1,2}$ and Zhi-Xi Wang ${ }^{1}$ \\ ${ }^{1}$ Department of Mathematics, Capital Normal University, Beijing 100037 \\ 2 Max-Planck-Institute for Mathematics in the Sciences, 04103 Leipzig
}

\begin{abstract}
We generalize the Schmidt-correlated states to multipartite systems. The related equivalence under SLOCC, the separability, entanglement witness, entanglement measures of negativity, concurrence and relative entropy are investigated in detail for the generalized Schmidt-correlated states.
\end{abstract}

Keywords: Schmidt-correlated state; separability; entanglement measure

\section{INTRODUCTION}

Quantum entanglement is of special importance in quantum information processing and responsible for many quantum tasks such as teleportation, dense coding, key distribution, error correction etc. [1]. Entanglement of bipartite states have been extensively studied. There have been many necessary or/and sufficient conditions on separability for certain states such as PPT, reduction, majorization, realignment etc. [2, 3, 4, 5, 6]. There are also some well defined measures of entanglement for bipartite states, e.g. entanglement of formation (EoF) [7, 8], concurrence [9], negativity [10] and relative entropy [11]. For two-qubit case EoF is a monotonically increasing function of concurrence and an elegant formula of concurrence was derived analytically by Wootters in [12]. For higher dimensional case, due to the extremizations involved in the calculation, only a few of explicit analytic formulae for EoF and concurrence have been found for some special symmetric states [13, 14], and attention has been payed to possible lower bounds of the EoF and concurrence e.g. [15, 16, 17].

Among the quantum states, the Schmidt-correlated (SC) states are of special properties. They are the mixtures of pure states, sharing the same Schmidt bases. It was first appeared in [18], named as maximally correlated state. For any classical measurement related to the $\mathrm{SC}$ states, two observers will always obtain the same result. Two SC states can always be optimally discriminated locally. The maximally entangled states (Bell state) can always be expressed in Schmidt correlated form. SC states naturally appear in a bipartite system dynamics with additive integrals of motion [19]. Hence, these states form an important class of mixed states from a quantum dynamical perspective. Bipartite SC states have been studied in [11, 20, 21, 22, 23]. The Relative Entropy of SC bipartite states has been 
investigated in [11, 20, 22]. The properties of negativity for SC states are studied in [23]. In this paper we generalize the Schmidt-correlated bipartite states to multipartite ones. We study the separability and entanglement of the multipartite SC states.

\section{DEFINITION}

We first give the definition of multipartite SC state in arbitrary dimension. We call a $k$-partite state $\rho$ in $C^{N} \otimes C^{N} \otimes \cdots \otimes C^{N}$ an SC state if it can be expressed as

$$
\rho=\sum_{m, n=0}^{N-1} a_{m n}|m \cdots m\rangle\langle n \cdots n|
$$

where $\sum_{m=0}^{N-1} a_{m m}=1$. (11) is a direct generalization of bipartite ones. If $A_{i}(1 \leq i \leq k)$ is the observer associated with the $i$-th sub-system, then $A_{1}, \cdots, A_{k}$ will always obtain the same result for any classical measurement.

It has been shown in [22] that for a general mixed state $\rho=\sum_{m, n=0}^{N-1} a_{m n}|m\rangle\langle n|$, there exists an ensemble of pure states $\left\{p_{i},\left|\Phi_{i}\right\rangle\right\}$ realizing $\rho$, where $\left|\Phi_{i}\right\rangle$ is of the form $\left|\Phi_{i}\right\rangle=$ $\sum_{m} \sqrt{a_{m m}} e^{i \Theta_{m}^{(i)}}|m\rangle$. This result can be easily generalized to multipartite case: the SC state (11) could be realized by an ensemble $\left\{p_{i},\left|\Phi_{i}\right\rangle\right\}$, where $\left|\Phi_{i}\right\rangle=\sum_{m} \sqrt{a_{m m}} e^{i \Theta_{m}^{(i)}}|m \cdots m\rangle$, with $a_{m m}$ given in (1). Furthermore, $\rho$ can only be realized by such ensembles. In fact, if there exists an ensemble $\left\{p_{i},\left|\Phi_{i}\right\rangle\right\}$ realizing $\rho$ such that $\left|\Phi_{i}\right\rangle=\sum_{m_{1}, \cdots, m_{k}} c_{m_{1}, \cdots, m_{k}}^{i}\left|m_{1}, \cdots, m_{k}\right\rangle$ for some $i$ and different $m_{1}, \cdots, m_{k}$, there must be an item $\left|m_{1}, \cdots, m_{k}\right\rangle\left\langle m_{1}, \cdots, m_{k}\right|$ in the presentation of $\rho$, so that $\rho$ is no longer of the form (1).

Let $\operatorname{GHZ}(k, N)$ denote the $k$-partite maximally entangled state

$$
\operatorname{GHZ}(k, N)=\frac{1}{\sqrt{N}}(|0 \cdots 0\rangle+|1 \cdots 1\rangle+\cdots+|N-1, \cdots, N-1\rangle) .
$$

Then $\left|\Phi_{i}\right\rangle$ is equivalent to either a fully separable state or $\operatorname{GHZ}(k, t)(0 \leq t \leq N)$ under stochastic local operation and classical communication (SLOCC) [24, 25]. Indeed, if $a_{m m}(0 \leq m \leq N-1)$ are all zero except one, then $\left|\Phi_{i}\right\rangle$ is fully separable. If not, let us 
suppose $a_{00}, \cdots, a_{(t-1),(t-1)}(1<t \leq N)$ are nonzero and the rest are zero. Let

$$
\begin{aligned}
& F=\left(\begin{array}{cccc}
\frac{1}{\left(\sqrt{t} \sqrt{a_{00}} e^{i \Theta_{0}^{(i)}}\right)^{\frac{1}{k}}} & 0 & \cdots & 0 \\
0 & \frac{1}{\left(\sqrt{t} \sqrt{a_{11}} e^{i \Theta_{1}^{(i)}}\right)^{\frac{1}{k}}} & \cdots & 0 \\
\cdots & \cdots & \cdots & \cdots \\
0 & 0 & \cdots & \frac{1}{\left(\sqrt{t} \sqrt{a_{t-1, t-1}} e^{i \Theta_{t-1}^{(i)}}\right)^{\frac{1}{k}}}
\end{array}\right) \\
& =\frac{1}{\left(\sqrt{t} \sqrt{a_{00}} e^{\left.i \Theta_{0}^{(i)}\right)^{\frac{1}{k}}}\right.}|0\rangle\left\langle 0\left|+\frac{1}{\left(\sqrt{t} \sqrt{a_{11}} e^{\left.i \Theta_{1}^{(i)}\right)^{\frac{1}{k}}}\right.}\right| 1\right\rangle\langle 1|+\cdots \\
& +\frac{1}{\left(\sqrt{t} \sqrt{a_{t-1, t-1}} e^{\left.i \Theta_{t-1}^{(i)}\right)^{\frac{1}{k}}}\right.}|t-1\rangle\langle t-1| .
\end{aligned}
$$

Obviously $F$ is invertible. We can get $\operatorname{GHZ}(k, t)=F \otimes F \otimes \cdots \otimes F\left|\Phi_{i}\right\rangle$.

In [26] it has been proven that if the partial inner product of a basis of any one of the subsystems with the state of a composite system gives a disentangled basis, then Schmidt decomposition for a tripartite system exists. This criterion can be generalized to multipartite states. We can see that $\left|\Phi_{i}\right\rangle$ are states which have Schmidt decompositions in a $k$-partite composite system.

\section{SEPARABILITY OF SCHMIDT-CORRELATED STATES}

The state (1) is fully separable if $\rho=\sum_{i} p_{i} \rho_{1}^{(i)} \otimes \rho_{2}^{(i)} \otimes \cdots \otimes \rho_{k}^{(i)}$, where $\sum_{i} p_{i}=1$, $\rho_{1}^{(i)}, \rho_{2}^{(i)}, \cdots, \rho_{k}^{(i)}$ are states in individual subsystems.

Proposition 1. State (1) is fully separable if and only if it is positive under partial transpositions with respect to some subsystems.

Proof. Without loss of generality, we choose $\rho^{T_{1}}$ as an example of partial transposition. The other cases are similar to $\rho^{T_{1}}$. The proof can be done along the same lines as calculation of negativity in [23]. In fact the eigenvalues of $\rho$ under partial transpositions with respect to any subsystems are the same to $\rho^{T_{1}}$. Hence $\rho$ is positive under some partial transpositions if and only if $\rho$ is positive under any partial transpositions. By carrying out some elementary transformations, $\rho^{T_{1}}=\sum_{m, n=0}^{N-1} a_{m n}|n m \cdots m\rangle\langle m n \cdots n|$, can be transformed into another matrix $\left(\rho^{T_{1}}\right)^{\prime}$,

$$
\left(\rho^{T_{1}}\right)^{\prime}=\left(\begin{array}{ccc}
A & 0 & 0 \\
0 & B & 0 \\
0 & 0 & C
\end{array}\right)
$$


where $A=\operatorname{diag}\left(a_{00}, a_{11}, \ldots, a_{N-1, N-1}\right)$,

$$
B=\left(\begin{array}{cccccccccc}
0 & a_{10} & 0 & 0 & \cdots & 0 & 0 & \cdots & 0 & 0 \\
a_{01} & 0 & 0 & 0 & \cdots & 0 & 0 & \cdots & 0 & 0 \\
0 & 0 & 0 & a_{20} & \cdots & 0 & 0 & \cdots & 0 & 0 \\
0 & 0 & a_{02} & 0 & \cdots & 0 & 0 & \cdots & 0 & 0 \\
\cdots & \cdots & \cdots & \cdots & \cdots & \cdots & \cdots & \cdots & \cdots & \cdots \\
0 & 0 & 0 & 0 & \cdots & 0 & a_{N-1,0} & \cdots & 0 & 0 \\
0 & 0 & 0 & 0 & \cdots & a_{0, N-1} & 0 & \cdots & 0 & 0 \\
\cdots & \cdots & \cdots & \cdots & \cdots & \cdots & \cdots & \cdots & \cdots & \cdots \\
0 & 0 & 0 & 0 & \cdots & 0 & 0 & \cdots & 0 & a_{N-1, N-2} \\
0 & 0 & 0 & 0 & \cdots & 0 & 0 & \cdots & a_{N-2, N-1} & 0
\end{array}\right),
$$

and $C$ is a zero matrix.

The nonzero eigenvalues of $\left(\rho^{T_{1}}\right)^{\prime}$ are $\lambda_{0}=a_{00}, \lambda_{1}=a_{11}, \cdots, \lambda_{N-1}=a_{N-1, N-1}, \lambda_{m n}^{ \pm}=$ $\pm\left|a_{m n}\right|, m<n, m, n=0, \cdots, N-1$, which are also the eigenvalues of $\rho^{T_{1}}$. Therefore $\rho^{T_{1}} \geq 0$ if and only if $\lambda_{m n}^{ \pm}=0$. In this case, the only nonzero eigenvalues of $\rho^{T_{1}}$ are $\lambda_{0}, \cdots, \lambda_{N-1}$ with respect to the eigenvectors $|0 \cdots 0\rangle, \cdots,|N-1, \cdots, N-1\rangle$. And $\rho=$ $\rho^{T_{1}}=a_{00}|0 \cdots 0\rangle\left\langle 0 \cdots 0\left|+\cdots+a_{N-1, N-1}\right| N-1, \cdots, N-1\right\rangle\langle N-1, \cdots, N-1|$. That is, $\rho$ is fully separable.

Conversely, if $\rho$ is fully separable, then $\rho$ is also bipartite separable in the partition $1 \mid 2 \cdots k, \rho=\sum_{i} q_{i} \rho_{1}^{(i)} \otimes \rho_{2 \cdots k}^{(i)}$. By Peres' criterion [2], we have $\rho^{T_{1}} \geq 0$.

Therefore for an SC state $\rho, \rho$ is fully separable if and only if it is positive with respect to some partial transposition, and $\rho$ is genuinely entangled if and only if it is not positive with respect to some partial transposition.

Entanglement witness is an operator that is designed for distinguishing between separable and entangled states [3, 27, 28]. A Hermitian operator $W$ is called an entanglement witness if it has a positive expectation value with respect to any separable state $\sigma, \operatorname{Tr}[W \sigma] \geq 0$, while there exists at least one entangled state $\rho$ such that $\operatorname{Tr}[W \rho]<0$. Next we construct entanglement witness for SC states.

Corollary 1. Let $\left|\Psi_{m n}\right\rangle$ be eigenvectors associated with the eigenvalues $\lambda_{m n}^{-}, m<n$, $m, n=0, \cdots, N-1$. Then $W=\sum_{m<n}\left(\left|\Psi_{m n}\right\rangle\left\langle\Psi_{m n}\right|\right)^{T_{1}}$ is an entanglement witness of $\rho$.

Proof. For any separable state $\sigma$, we have

$$
\operatorname{Tr}[W \sigma]=\sum_{m<n} \operatorname{Tr}\left[\left(\left|\Psi_{m n}\right\rangle\left\langle\Psi_{m n}\right|\right)^{T_{1}} \sigma\right]=\sum_{m<n} \operatorname{Tr}\left[\left|\Psi_{m n}\right\rangle\left\langle\Psi_{m n}\right| \sigma^{T_{1}}\right] \geq 0
$$


as $\sigma^{T_{1}} \geq 0$. For the entangled SC state $\rho$, we get

$$
\operatorname{Tr}[W \rho]=\sum_{m<n} \operatorname{Tr}\left[\left(\left|\Psi_{m n}\right\rangle\left\langle\Psi_{m n}\right|\right)^{T_{1}} \rho\right]=\sum_{m<n} \operatorname{Tr}\left[\left|\Psi_{m n}\right\rangle\left\langle\Psi_{m n}\right| \rho^{T_{1}}\right]=\sum_{m<n} \lambda_{m n}^{-}<0 .
$$

Therefore $W$ is a witness for $\rho$.

Any bipartite state $\rho$ in $V_{1} \otimes V_{2}$ can be written in Bloch representation [29]. Let $\lambda_{i}(0 \leq$ $\left.i \leq N^{2}-2\right)$ be the generators of $S U(N), 0 \leq j<k \leq N-1$,

$$
\begin{gathered}
\lambda_{i}=\sqrt{\frac{2}{(i+1)(i+2)}}\left(\sum_{a=0}^{i}|a\rangle\langle a|-(i+1)| i+1\rangle\langle i+1|\right), \quad i=0, \cdots, N-2, \\
\lambda_{i}=|j\rangle\langle k|+| k\rangle\langle j|, \quad i=N-1, \cdots, \frac{(N+2)(N-1)}{2}-1, \\
\lambda_{i}=-i(|j\rangle\langle k|-| k\rangle\langle j|), \quad i=\frac{(N+2)(N-1)}{2}, \cdots, N^{2}-2 .
\end{gathered}
$$

Any bipartite state $\rho$ can be written as

$$
\rho=\frac{1}{M N}\left(I_{M} \otimes I_{N}+r_{i} \lambda_{i} \otimes I_{N}+s_{j} I_{M} \otimes \lambda_{j}+t_{i j} \lambda_{i} \otimes \lambda_{j}\right),
$$

where $M=\operatorname{dim} V_{1}, N=\operatorname{dim} V_{2}, r_{i}=\frac{M}{2} \operatorname{Tr}\left(\rho \lambda_{i} \otimes I_{N}\right), s_{j}=\frac{N}{2} \operatorname{Tr}\left(\rho I_{M} \otimes \lambda_{j}\right), t_{i j}=\frac{M N}{4^{4}} \operatorname{Tr}\left(\rho \lambda_{i} \otimes\right.$ $\left.\lambda_{j}\right)$. A pure bipartite state is separable if and only if $t_{i j}=r_{i} s_{j}$ for any $i$ and $j[29]$.

For a multipartite SC state $\rho$, we express it in Bloch representation as a bipartite decomposition. Assuming that a given $k$-partite SC state is divided in to two subsystems, the first one consisting of $l$ parties and the second one of $(k-l)$ parties. Thus the subsystems are defined on $N^{l}$-dimensional and $N^{k-l}$-dimensional Hilbert spaces, respectively. This means in turn that to describe such a state in the Bloch representation one can take generators of $S U\left(N^{l}\right)$ and $S U\left(N^{k-l}\right)$ as bases in respective subsystems. By calculation we find $r_{i}=0$ for $i \geq N^{l}-1, s_{i}=0$ for $i \geq N^{k-l}-1, t_{i j}=0$ for $i \geq N^{l}-1,0 \leq j \leq N^{k-l}-2$, or $0 \leq i \leq N^{l}-2, j \geq N^{k-l}-1$. According to our Proposition 1, (11) is separable if and only if $\rho=\sum_{m=0}^{N-1} a_{m m}|m \cdots m\rangle\langle m \cdots m|$. We have $t_{i j}=0$ for a separable SC state, where $i \geq N^{l}-1, j \geq N^{k-l}-1$. Conversely, if $t_{i j}=0$ for all $i \geq N^{l}-1, j \geq N^{k-l}-1$, then $a_{m n}=0$ for $m \neq n$. Hence $\rho$ is fully separable. Consequently, we get

Corollary 2. A multipartite SC state, expressed in Bloch representation as a bipartite decomposition, is fully separable if and only if $t_{i j}=0$ holds for $i \geq N^{l}-1, j \geq N^{k-l}-1$.

Another complementary operational separability criterion is called realignment criterion [5, 6] which detects even bound entangled states. It says that for any separable state the realigned matrix $R(\rho)$ of $\rho$ satisfies $\|R(\rho)\| \leq 1$, where $\|\rho\| \equiv \operatorname{tr} \sqrt{\rho^{\dagger} \rho}, R(\rho)$ is given by $R(\rho)_{i j, k l}=\rho_{i k, j l}$ with $i$ and $j$ the row and column indices for the first subsystem and $k$ and $l$ the indices for the second subsystem. For multipartite SC states, let us first view them as bipartite states in $\mathbb{C}^{N} \otimes \mathbb{C}^{M}$. 
Proposition 2. For any SC state $\rho$ in $V_{1} \otimes V_{2} \otimes \cdots \otimes V_{k}, \operatorname{dim} V_{i}=N(1 \leq i \leq k)$, we have $1 \leq\|R(\rho)\| \leq N$. A state $\rho$ is fully separable (resp. maximally entangled) if and only if $\|R(\rho)\|=1$ (resp. $\|R(\rho)\|=N)$.

Proof. For multipartite SC states (1), we have that $R(\rho)$ is a diagonal matrix with diagonal entries $a_{m n}$ and some zeros. Thus

$$
\|R(\rho)\|=\sum_{m, n=0}^{N-1}\left|a_{m n}\right| \leq \sum_{m, n=0}^{N-1} \sqrt{a_{m m} a_{n n}} \leq \sum_{m, n=0}^{N-1} \frac{1}{2}\left(a_{m m}+a_{n n}\right)=N .
$$

The first inequality follows from the positivity of the density operator and the second one from the inequality of geometric and arithmetic means.

Here $\|R(\rho)\|=1$ implies $a_{m n}=0$ for $m \neq n, m, n=0, \cdots, N-1$. Therefore in this case $\rho$ is fully separable. When $\|R(\rho)\|=N$, we obtain $a_{m n}=\frac{1}{N}$ for all $m, n=0,1, \cdots, N-1$, i.e. $\rho$ is $\operatorname{GHZ}(k, N)$. Hence $\rho$ is maximally entangled in any bipartite decompositions.

\section{ENTANGLEMENT OF SCHMIDT-CORRELATED STATES}

We now calculate some measures of entanglement for multipartite SC states.

\section{A. Negativity}

One entanglement measure for bipartite states defined in [10] is the negativity, $N(\rho)=$ $\frac{\left\|\rho^{T_{1}}\right\|-1}{2}$, which corresponds to the absolute value of the sum of negative eigenvalues of $\rho^{T_{1}}$. Now let's have a look at the negativity of SC states in a bipartite decomposition.

For any SC state $\rho$ in $V_{1} \otimes V_{2} \otimes \cdots \otimes V_{k}, \operatorname{dim} V_{i}=N(1 \leq i \leq k)$, it is straightforward to verify that $N(\rho)=\frac{1}{2} \sum_{m \neq n}\left|a_{m n}\right|=\frac{\|R(\rho)\|-1}{2}$. From Proposition 2 we have $0 \leq N(\rho) \leq \frac{N-1}{2}$ associated with any partial transpositions. If $N(\rho)=0$ for some partial transpositions, then $\rho$ is fully separable. If $N(\rho)=\frac{N-1}{2}$ with respect to some partial transpositions, then $\rho$ is the maximally entangled pure GHZ state.

Indeed, for any $|\Psi\rangle=\sum_{\alpha} c_{\alpha} e_{\alpha}^{\prime} \otimes e_{\alpha}^{\prime \prime}, N(\rho)=\frac{1}{2}\left[\left(\sum_{\alpha} c_{\alpha}\right)^{2}-1\right]$ [10]. As an example for the maximally entangled state $\operatorname{GHZ}(2, N)=\frac{1}{\sqrt{N}}(|00\rangle+|11\rangle+\cdots+|N-1, N-1\rangle)$, we have $N(\mathrm{GHZ}(2, N))=\frac{N-1}{2}$.

Since SC states are either genuinely entangled or fully separable, we know that if $N(\rho) \neq$ 0 , then $\rho$ must be genuinely entangled. But it may be not maximally entangled. For instance, for states in $\mathbb{C}^{2} \otimes \mathbb{C}^{2} \otimes \mathbb{C}^{2}: \rho=\frac{2}{3}\left[\frac{1}{2}(|000\rangle+|111\rangle)(\langle 000|+\langle 111|)\right]+\frac{1}{3}|000\rangle\langle 000|$ and $\operatorname{GHZ}(3,2)=\frac{1}{2}(|000\rangle+|111\rangle)(\langle 000|+\langle 111|)$, we have $N(\rho)=1 / 3$ and $N(\operatorname{GHZ}(3,2))=1 / 2$ respectively. $\rho$ is genuinely entangled but not maximally entangled. 


\section{B. Concurrence}

The concurrence for a bipartite pure state $|\Psi\rangle$ is defined by $C(|\Psi\rangle)=\sqrt{2\left(1-\operatorname{Tr} \rho_{1}^{2}\right)}$, where the reduced density matrix $\rho_{1}$ is given by $\rho_{1}=\operatorname{Tr}_{2}(|\Psi\rangle\langle\Psi|)$. The concurrence is then extended to mixed states $\rho$ by the convex roof, $C(\rho) \equiv \min _{\left\{p_{i},\left|\Psi_{i}\right\rangle\right\}} \sum_{i} p_{i} C\left(\left|\Psi_{i}\right\rangle\right)$, for all possible ensemble realizations $\rho=\sum_{i} p_{i}\left|\Psi_{i}\right\rangle\left\langle\Psi_{i}\right|$, where $p_{i} \geq 0$ and $\sum_{i} p_{i}=1$. A state $\rho$ is separable if and only if $C(\rho)=0$.

For a multipartite $\mathrm{SC}$ state $\rho$,

$$
\rho=\sum_{m, n=0}^{N-1} a_{m n}|m \cdots m\rangle\left\langle n \cdots n\left|=\sum_{i} p_{i}\right| \Psi_{i}\right\rangle\left\langle\Psi_{i}\right|,
$$

where, as discussed in section II, $\left|\Psi_{i}\right\rangle$ takes the form $\left|\Psi_{i}\right\rangle=\sum_{m} c_{m}^{(i)}|m \cdots m\rangle, \sum_{m}\left|c_{m}^{(i)}\right|^{2}=1$, $a_{m n}=\sum_{i} p_{i} c_{m}^{(i)} c_{n}^{(i) *}$. We get that the concurrences of $\left|\Psi_{i}\right\rangle$ are the same for all reduced density matrices in bipartite decompositions. Let us choose $\rho_{1}=\operatorname{Tr}_{2 \ldots k}\left(\left|\Psi_{i}\right\rangle\left\langle\Psi_{i}\right|\right)$ as an example. We have $\rho_{1}=\sum_{m}\left|c_{m}^{(i)}\right|^{2}|m\rangle\langle m|$,

$$
\begin{aligned}
C\left(\left|\Psi_{i}\right\rangle\right) & =\sqrt{2\left(1-\sum_{m}\left|c_{m}^{(i)}\right|^{4}\right)}, \\
C(\rho) & =\min _{\left\{p_{i},\left|\Psi_{i}\right\rangle\right\}} \sum_{i} p_{i} \sqrt{2\left(1-\sum_{m}\left|c_{m}^{(i)}\right|^{4}\right)}=2 \min _{\left\{p_{i},\left|\Psi_{i}\right\rangle\right\}} \sum_{i} p_{i} \sqrt{\sum_{m<n}\left|c_{m}^{(i)} c_{n}^{(i)}\right|^{2}} .
\end{aligned}
$$

Taking into account that $\sum_{m}\left|c_{m}^{(i)}\right|^{2}=1$, we have $0 \leq C(\rho) \leq \sqrt{2\left(1-\frac{1}{N}\right)}$. For the state $\operatorname{GHZ}(k, N)$, one has

$$
C(\operatorname{GHZ}(k, N))=\sqrt{2\left(1-\sum_{m=0}^{N-1} \frac{1}{N^{2}}\right)}=\sqrt{2\left(1-\frac{1}{N}\right)} .
$$

In [15] an analytical lower bound for the concurrence of bipartite quantum states in arbitrary dimension has been derived, which is exact for some mixed quantum states. For $N$-dimensional SC states $\rho$, the result is reduced to be $C(\rho) \geq \frac{2 \sqrt{2}}{\sqrt{N(N-1)}} N(\rho)$. In particular, for multi-qubit $(N=2) \mathrm{SC}$ states $\rho=\sum_{i} p_{i}\left|\Phi_{i}\right\rangle\left\langle\Phi_{i}\right|$, if $\left|\Phi_{i}\right\rangle=\alpha_{i}|0 \cdots 0\rangle+\beta_{i} e^{i \theta}|1 \cdots 1\rangle$, where $\alpha_{i}, \beta_{i} \geq 0$ and $0 \leq \theta \leq 2 \pi$, then $C(\rho)=2 \min _{\left.\left\{p_{i}, \Psi_{i}\right\rangle\right\}} \sum_{i} p_{i} \alpha_{i} \beta_{i}=2 N(\rho)$. Namely if all the pure states $\left|\Phi_{i}\right\rangle$ in the decomposition share the same relative phase $e^{i \theta}$, then the equality holds, $C(\rho)=2 N(\rho)=\|R(\rho)\|-1$. Moreover, for pure SC qubit states, we have $C(\rho)=2 N(\rho)=\|R(\rho)\|-1$. For example, for $|\Psi\rangle=\sqrt{\frac{1}{3}}|0 \cdots 0\rangle+\sqrt{\frac{2}{3}}|1 \cdots 1\rangle$, we have $C(|\Psi\rangle)=2 N(|\Psi\rangle)=\| R(|\Psi\rangle) \|-1=\frac{2 \sqrt{2}}{3}$. 
Concerning the concurrence for multipartite states, instead of bipartite decompositions, there is also a generalized version:

$$
C(|\Psi\rangle)=\sqrt{k-\operatorname{Tr} \rho_{1}^{2}-\operatorname{Tr} \rho_{2}^{2}-\cdots-\operatorname{Tr} \rho_{k}^{2}}
$$

where the reduced density matrix $\rho_{i}$ is given by $\rho_{i}=\operatorname{Tr}_{1, \cdots, i-1, i+1, \cdots, k}(|\Psi\rangle\langle\Psi|), i=1,2, \cdots, k$. Extending to mixed states $\rho$,

$$
C(\rho) \equiv \min _{\left\{p_{i},\left|\Psi_{i}\right\rangle\right\}} \sum_{i} p_{i} C\left(\left|\Psi_{i}\right\rangle\right)
$$

for all possible ensemble realizations $\rho=\sum_{i} p_{i}\left|\Psi_{i}\right\rangle\left\langle\Psi_{i}\right|$. Similarly we can get $0 \leq C(\rho) \leq$ $\sqrt{k\left(1-\frac{1}{N}\right)}$ from Lagrange multipliers method. Applying to the state $\operatorname{GHZ}(k, N)$, we have

$$
C(\operatorname{GHZ}(k, N))=\sqrt{k\left(1-\frac{1}{N}\right)} .
$$

\section{Relative Entropy}

Relative Entropy is a well defined measure for multipartite states. For a density ma$\operatorname{trix} \rho, E(\rho)=\min _{\sigma \in D} S(\rho \| \sigma)=\min _{\sigma \in D} \operatorname{Tr}[\rho \log \rho-\rho \log \sigma]$, where $D$ is the set of all fully separable states [11]. However, it is not easy to find the optimal separable state $\sigma^{*}$ such that $E(\rho)=\min _{\sigma \in D} S(\rho \| \sigma)=S\left(\rho \| \sigma^{*}\right)$. It has been proven that $\sigma^{*}=\sum_{m} a_{m m}|m m\rangle\langle m m|$ is PPT optimal for bipartite state $\rho=\sum_{m n} a_{m n}|m m\rangle\langle n n|$ [20], that is, $S\left(\rho \| \sigma^{*}\right)=\min _{\sigma^{\prime} \in P} S\left(\rho \| \sigma^{\prime}\right)$, where $P$ is the set of all PPT states.

For a $k$-partite SC state $\rho=\sum_{m n} a_{m n}|m \cdots m\rangle\langle n \cdots n|, E(\rho)=\min _{\sigma \in D} S(\rho \| \sigma)=S(\rho \|$ $\left.\sigma^{*}\right)$, where $\sigma^{*}=\sum_{m} a_{m m}|m \cdots m\rangle\langle m \cdots m|$, and $D$ is the set of all fully separable states. We consider a $k$-partite SC state as a bipartite SC state, for example, the first subsystem and the rest subsystems, $\rho=\sum_{m n} a_{m n}(|m\rangle \otimes|m \cdots m\rangle)(\langle n| \otimes\langle n \cdots n|)$. Then according to [20], $S\left(\rho \| \sigma^{*}\right)=\min _{\sigma^{\prime} \in P} S\left(\rho \| \sigma^{\prime}\right)$, where $P$ is the set of all PPT states. Since fully separable states are all positive under partial transpose with respect to any subsystems, we have $\min _{\sigma \in D} S(\rho \| \sigma) \geq \min _{\sigma^{\prime} \in P} S\left(\rho \| \sigma^{\prime}\right)$. Because $\sigma^{*}$ is separable, we get the formula $E(\rho)=\min _{\sigma \in D} S(\rho \| \sigma)=S\left(\rho \| \sigma^{*}\right)$ also for multipartite SC states.

For the state $\operatorname{GHZ}(k, N)$, the optimal fully separable state is just

$$
\sigma^{*}=\frac{1}{N} \sum_{i=0}^{N-1}|i i \cdots i\rangle\langle i i \cdots i| .
$$

And $E(\operatorname{GHZ}(k, N))=S\left(\operatorname{GHZ}(k, N) \| \sigma^{*}\right)=\operatorname{Tr}\left[\rho \log \rho-\rho \log \sigma^{*}\right]=\log N$.

By calculating three kinds of entanglement measures for SC states, we can see that the entanglement of multipartite maximally entangled states is independent on the number of subsystems $k$. The entanglement is only related to the dimensions of the subsystems. 


\section{CONCLUSIONS}

We have investigated a special kind of multipartite states named SC states. The sufficient and necessary conditions of separability for these states have been studied. We have also calculated the negativity, concurrence and relative entropy of SC states. By calculating the $N(\rho), C(\rho)$ or $E(\rho)$, the entanglements of any two SC states can be compared. Moreover, like bipartite SC states that naturally appear in dynamics with additive integrals of motion [19], the multipartite SC states, which have always bipartite decompositions, would also form an important class of mixed states from a quantum dynamical perspective.

[1] M.A. Nielsen and I.L. Chuang, Quantum Computation and Quantum Information (Cambridge Univesity Press, Cambridge, 2000).

[2] A. Peres, Phys. Rev. Lett. 77, 1413(1996).

[3] M. Horodecki, P. Horodecki and R. Horodecki, Phys. Lett. A 223, 1(1996).

[4] M. A. Nielsen and J. Kempe, Phys. Rev. Lett. 86, 5184(2001).

[5] O. Rudolph, Quantum Inf. Proc. 4, 219(2005).

[6] K. Chen and L.A. Wu. Quantum Inf. Comput. 3, 193(2003).

[7] C.H. Bennett, D.P. DiVincenzo, J.A. Smolin, and W.K. Wootters, Phys. Rev. A 54, 3824(1996).

[8] M. Horodecki, Quantum Inf. Comp. 1, 3(2001);

D. Bruß, J. Math. Phys. 43, 4237(2002);

M.B. Plenio and S. Virmani, Quantum Inf. Comp. 7, 1(2007).

[9] A. Uhlmann, Phys. Rev. A 62, 032307(2000);

P. Rungta, V. Buzek, C.M. Caves, M. Hillery and G.J. Milburn, Phys. Rev. A 64, 042315(2001);

S. Albeverio and S. M. Fei, J. Opt. B: Quantum Semiclass. Opt. 3, 223(2001).

[10] G. Vidal and R. F. Werner, Phys. Rev. A 65, 032314(2002).

[11] V. Vedral and M.B. Pleniio, Phys Rev A 57, 1619(1998).

[12] W.K. Wootters, Phys. Rev. Lett. 80, 2245(1998).

[13] B.M. Terhal, K. Gerd and K.G.H. Vollbrecht, Phys. Rev. Lett. 85, 2625(2000).

S.M. Fei, J. Jost, X.Q. Li-Jost and G.F. Wang, Phys. Lett. A 310, 333(2003). 
S.M. Fei, Z.X. Wang and H. Zhao, Phys. Lett. A 329, 414(2004).

[14] P. Rungta and C.M. Caves, Phys. Rev. A 67, 012307(2003).

[15] K. Chen, S. Albeverio, and S. M. Fei, Phys. Rev. Lett. 95, 040504(2005).

[16] K. Chen, S. Albeverio, and S. M. Fei, Phys. Rev. Lett. 95, 210501(2005).

[17] Heinz-Peter Breuer, J. Phys. A: Math. Gen. 39, 11847(2006)

[18] E.M. Rains, Phys. Rev. A 60, 179(1999).

[19] M. Khasin, R. Kosloff, Phys. Rev. A 76, 012304(2007).

[20] E.M. Rains, Phys. Rev. A 60, 1(1999) .

[21] S. Virmani, M.F. Sacchi, M.B. Plenio, D. Markham, Phys. Lett. A 288, 62(2001).

[22] Y.X. Chen and D. Yang, Quantum Inf. Proc. 1, 389(2002).

[23] M. Khasin, R. Kosloff and D. Steinitz, Phys. Rev. A 75, 052325(2007).

[24] F. Pan and G.Y. Lu, Int. J. Mod. Phys. B 20, 1333(2006).

[25] L. Lamata, J. León, D. Salgado and E. Solano, Phys. Rev. A 74, 052336(2006).

[26] A.K. Pati, Phys. Lett. A 278, 118(2000).

[27] B. M. Terhal, Phys. Lett. A 271, 319(2000).

[28] P. Hyllus, O. Gühne, D. Bruß and M. Lewenstein, Phys. Rev. A 72, 012321(2005).

[29] J.I. de Vicente, Quantum Inf. Comput. 7, 624(2007). 